\title{
77. NUEVAS APORTACIONES A LA FLORA DE LA PROVINCIA DE ALICANTE
}

\author{
José Gabriel SEGARRA MORAGUES
}

\begin{abstract}
New floristic records for Alicante province.
Palabras clave. Flora, Corología, Alicante, Comunidad Valenciana, España.
\end{abstract}

Key words. Flora, Corology, Alicante, Valencian Community, Spain.

El presente trabajo incluye diez hallazgos interesantes para el levante peninsular. De ellos, una resulta una nueva aportación a la flora de la Comunidad Valenciana, siete son novedades para la provincia de Alicante y dos se citan por segunda vez para la provincia, lo cual supone una ampliación de su área de distribución conocida hasta el momento. Todas ellas, son el resultado de un estudio realizado sobre la flora del término municipal de Xàbia. Se trata de una localidad costera del noreste de la provincia de Alicante, caracterizada por presentar afloramientos de rocas calizas pertenecientes a las unidades externas de las cordilleras Béticas (Estévez \& Soria, 1991) y un encuadre bioclimatológico en el piso termomediterráneo de ombroclima seco.

\section{Bifora testiculata (L.) Roth.}

ALICANTE: Xàbia, La Granadella, 31SBC5590, 180m, campos de cereal, 12-IV-1997, J.G. Segarra, VAB 981334.

Taxon distribuido por toda la cuenca mediterránea y que esta presente en gran parte de la Península Ibérica. Recientemente ha sido citada en la provincia de Alicante por De la Torre \& Vicedo (1998). Esta recolección representa una ampliación de área dentro de la provincia.

Diplotaxis muralis (L.) DC.

ALICANTE: Xàbia, Montañar, 31 SBC5794, 3 $\mathrm{m}$, terrenos baldíos, 24-V-1997. J.G. Segarra, VAB 981330.
Taxon de distribución Holártica, relegado, en la Península Ibérica, a su mitad oriental, desde Cataluña hasta Valencia (Bolòs \& Vigo, 1990). Hasta la revisión del género para Flora Iberica, se creía presente en la provincia de Alicante; sin embargo, estas citas correspondían a errores en la determinación, debido a confusiones con otras especies del género presentes en el área (MartinezLaborde, 1993). Dado que estas citas previas para Alicante han sido invalidadas, nuestro hallazgo resulta una novedad para la provincia de Alicante.

Juncus striatus Schousboe

ALICANTE: Xàbia, Barranqueres, 31SBC4896, $60 \mathrm{~m}$, barranco húmedo, 28-VI- 96. J.G. Segarra, VAB 981337.

Taxon distribuido por toda la región Mediterránea. En la Comunidad Valenciana, se conoce de la provincia de Valencia, donde es calificada como muy rara (Mateo \& Crespo, 1998), no conocemos citas previas para la provincia de Alicante.

Lathyrus ochrus (L.) DC.

ALICANTE: Xàbia, Barranqueres, 31 SBC5196, $60 \mathrm{~m}$, herbazales nitrófilos húmedos, 26-IV-1997. J.G. Segarra, VAB 981331.

Taxon ampliamente distribuido por toda la cuenca Mediterránea. En la Península Ibérica, resulta más abundante en su parte sudoccidental. En el Este de la Península, es conocida de Almería, Barcelona, Gerona y Murcia (Gallego, 1999). Recientemente ha sido citada de una localidad alicantina por Soler et al. (1995), constituyendo ésta, junto con nuestra recolección, las dos únicas localidades conocidas hasta el momento en la Comunidad Valenciana. 
Medicago ciliaris (L.) All.

ALICANTE: Xàbia, Desembocadura río Gorgos, 31SBC5196, $60 \mathrm{~m}$, herbazales salinos nitrófilos y húmedos, 09-XI-1996. J.G. Segarra, VAB 981329.

Taxon distribuido por la cuenca mediterránea. En la parte oriental de la Península Ibérica, su distribución resulta bastante puntual, con algunas localidades de Albacete (Herranz \& Gómez-Campo, 1986), Murcia y de Cataluña (Bolòs \& Vigo, 1984), donde se la califica de muy rara. No conocemos citas anteriores en la provincia de Alicante.

\section{Medicago scutellata (L.) Miller}

ALICANTE: Xàbia, Barranqueres, 31SBC5196, $60 \mathrm{~m}$, herbazales nitrófilos húmedos, 09-XI-1996. J.G. Segarra, VAB 981329.

Taxon distribuido por toda la cuenca mediterránea. En la parte oriental de la Península Ibérica, su distribución queda relegada a algunas localidades de Murcia y de Cataluña, donde se la califica de muy rara (Bolòs \& Vigo, 1984). En la Comunidad Valenciana, solamente se conocía anteriormente de la provincia de Castellón (Mateo \& Crespo, 1995, 1998).

Ranunculus peltatus Schrank subsp. baudotii (Godron) C.D.K. Cook

ALICANTE: Xàbia, Cauce del río Gorgos, 31SBC5295, $60 \mathrm{~m}$, charcas temporales, 26-IV-1997. J.G. Segarra, VAB 981500.

Taxon disperso por la mayor parte de la Península Ibérica (Bolòs \& Vigo, 1984, Cook 1986). En la Comunidad Valenciana se encuentra de manera abundante en los cultivos de arroz de la provincia de Valencia (Mateo \& Figuerola, 1987). No se conocen citas anteriores para la provincia de Alicante, y su presencia por el momento, resulta muy esporádica, probablemente, se deba al hecho de que la mayor parte de las zonas húmedas de esta provincia, presentan un elevado grado de salinidad, donde, por lo visto no es capaz de desarrollarse.

\section{Serapias lingua $\mathrm{L}$.}

ALICANTE: Xàbia, La Guardia, 31SBC5791, 170 m, Pastizal , 26-IV-1997. J.G. Segarra, VAB 981328.

Taxon de distribución mediterránea, conocida en la Comunidad Valenciana de la provincia de Valencia, en la que se la califica de muy rara (Mateo \& Crespo, 1995, 1998). No conocemos citas previas para la provincia de Alicante.

\section{Silene muscipula $\mathrm{L}$.}

ALICANTE: Xàbia, Cansalades, 31SBC5392, 100 m, Campo de cereal, 26-IV-1997. J.G. Segarra, VAB 982197

Taxon distribuido por toda la cuenca mediterránea (Bolòs \& Vigo, 1990). Repartida por casi toda la Península Ibérica, a excepción de la zona noroccidental y presente en la Comunidad Valenciana, en las provincias de Castellón y Valencia (Talavera, 1990). Hasta el momento no se conocía ninguna localidad en Alicante.

\section{Trifolium maritimum Huds.}

ALICANTE: Xàbia, Saladar, 31SBC5695, 2 m, Herbazales húmedos salinos, 12-IV-1997. J.G. Segarra, VAB 981338.

Taxon distribuido por la cuenca mediterránea y parte de la costa atlántica europea. En la Península Ibérica resulta más abundante en su mitad occidental, (Bolòs \& Vigo, 1984; Valdés et al. 1987). En la parte oriental, su distribución queda relegada a algunas localidades en las costas catalanas, en las que se la califica de rara (Bolòs \& Vigo, 1984). No se conocen citas previas en la Comunidad Valenciana.

AGRADECIMIENTOS: El autor quiere expresar su agradecimiento a Carlos Fabregat por la confirmación del ejemplar de Diplotaxis muralis y a la Dra. Isabel Mateu Andrés por la revisión crítica del manuscrito.

\section{BIBLIOGRAFÍA}

BOLÒS, O. y J. VIGO -1984- Flora dels Països Catalans I. Ed Barcino, Barcelona. 736 p.p.

BOLÒS, O. y J. VIGO -1990-Flora dels Països Catalans II. Ed Barcino, Barcelona. 921 p.p.

COOK, C.D.K. -1986- Ranunculus L. (subgen. Batrachium) en Castroviejo, S et al. (éds.). Flora Iberica I: Lycpodiaceae-Papaveraceae. Real Jardín Botánico, C.S.I.C. Madrid.

DE LA TORRE, A. \& M. VICEDO -1998- Fragmenta Chorologica Occidentalia 6621-6624. Anales Jard. Bot. Madrid 56(1): 143.

ESTÉVEZ, A. y J. SORIA -1991- en Stübing, G 
(Coord.) Estudio multidisciplinar del Parque Natural del Montgó (Alicante). Consellería d’Administració Pública. Agéncia del Medi Ambient. 283 p.p.

GALLEGO, M.J. -1999- Lathyrus L. en Castroviejo, $\mathrm{S}$ et al. (éds.). Flora Iberica VII:Leguminosae (partim). Real Jardín Botánico, C.S.I.C. Madrid. HERRANZ, J.M. y C. GÓMEZ-CAMPO -1986Contribución al conocimiento de la flora y vegetación de la comarca de Alcaráz (Albacete). Caja de Ahorros de Albacete. 279 p.p.

MARTINEZ-LABORDE, J.B. -1993- DC. en Castroviejo, S. et al. (éds) Flora Iberica IV: Cruciferae-Monotropaceae. Real Jardín Botánico, C.S.I.C. Madrid.

MATEO, G. y M.B. CRESPO -1995- Flora abreviada de la Comunidad Valenciana. Ed. Gamma. 483 p.p.

MATEO, G. y M.B. CRESPO - 1998- Manual para la determinación de la flora valenciana. Flora Montibérica: Monografías 3:1-495.

MATEO, G. y R. FIGUEROLA -1987- Flora analítica de la Provincia de Valencia. Institució Valenciana d’Estudis i Investigació. Edicións Alfons el Magnànim. IAM 14: 1-386
RIGUAL, A. -1972- Flora y vegetación de la provincia de Alicante. Instituto de Estudios Alicantinos. Alicante.

SOLER, J.X.; B. ROCHET y G. MATEO -1995Fragmenta Chorologica Occidentalia 54795509. Anales Jard. Bot. Madrid 53(1): 113-114.

TALAVERA, S. -1990- Silene L. in Castroviejo, S. et al. (éds); 1990-Flora Iberica II: PlatanaceaePlumbaginaceae (partim). Real Jardín Botánico, C.S.I.C. Madrid.

VALDÉS, B., S. TALAVERA, y E. FERNÁNDEZGALIANO -1987- Flora vascular de Andalucía Occidental, Vol. 2. Ed. Ketres. Barcelona.

Aceptado para su publicación en Octubre de 1999

Dirección del autor. Departamento de Biología Vegetal, U.D. Botánica, Facultad de Ciencias Biológicas, Universitat de València. Dr. Moliner, 50. 46100 Burjassot. (Valencia).

\title{
78. REFERENCIAS COROLÓGICAS DE PLANTAS VASCULARES EN EL NW IBÉRICO
}

\author{
Raquel ALONSO REDONDO, María José LÓPEZ PACHECO, \\ Emilio PUENTE GARCÍA y Ángel PENAS MERINO
}

Geographical distribution references of vascular plants for NW Spain

Palabras clave. Plantas vasculares, corología, NW España.

Key words. Vascular plants, geographical distribution, NW Spain.

En el presente trabajo ofrecemos una relación de plantas herborizadas en la provincia de León, señalando con un asterisco (*) aquellas que son novedad para dicha provincia. 